\title{
Anti-demodectic effects of okra eyelid patch in Demodex blepharitis compared with tea tree oil
}

\author{
WENTING LIU ${ }^{1,2}$ and LAN GONG ${ }^{1,2}$ \\ ${ }^{1}$ Department of Ophthalmology and Vision Science, The Eye, Ear, Nose and Throat Hospital of Fudan University; \\ ${ }^{2}$ NHC Key Laboratory of Myopia, Laboratory of Myopia, Chinese Academy of \\ Medical Sciences, Fudan University, Shanghai 200031, P.R. China
}

Received January 7, 2020; Accepted January 18, 2021

DOI: $10.3892 /$ etm.2021.9769

\begin{abstract}
Demodex infection gradually develops to Demodex blepharitis, which is characterized as chronic inflammation of the eyelid and meibomian gland (MG) and ultimately leads to MG dysfunction. In the present prospective study, the anti-demodectic effects of an okra eyelid patch in patients Demodex blepharitis were investigated. A total of 52 patients with Demodex blepharitis with ocular discomfort were recruited. Patients were randomized to receive either an okra eyelid patch treatment (treatment group, $n=27$ ) or tea tree oil (TTO) eye care patch treatment (control group, $n=25$ ) for three months. The Demodex count, the ocular surface disease index (OSDI) score, MG expressibility (MGE) and meibum quality, Schirmer I test (SIT), tear break-up time (TBUT) and corneal fluorescein staining (CFS) were determined prior to treatment and after 1 and 3 months of treatment. Changes in the parameters were compared between the treatment group and control group after 1 and 3 months of treatment. The average survival time in the okra group was $115.25 \pm 11.87 \mathrm{~min}$, which was significantly lower compared with the average ST of $378.75 \pm 37.94 \mathrm{~min}$ in the blank group $(\mathrm{P}<0.01)$. After 3 months of okra eyelid patch treatment, the Demodex count was significantly reduced from $10.15 \pm 4.53$ to $1.30 \pm 1.41(\mathrm{P}<0.01)$ and the OSDI score of the patients was reduced by $16.84 \pm 10.17(\mathrm{P}<0.01)$. There was no significant difference in the Demodex count $(\mathrm{P}=0.716)$ and OSDI $(\mathrm{P}=0.873)$ between the treatment and control groups. The
\end{abstract}

Correspondence to: Professor Lan Gong, Department of Ophthalmology and Vision Science, The Eye, Ear, Nose and Throat Hospital of Fudan University, 83 Fenyang Road, Shanghai 200031, P.R. China

E-mail: 13501798683@139.com

Abbreviations: CFS, corneal fluorescein staining; CD, cylindrical dandruff; MGE, meibomian gland expressibility; OSDI, ocular surface disease index; SIT, Schirmer I test; TTO, tea tree oil; TBUT, tear break-up time

Key words: okra, Demodex blepharitis, ocular surface, anti-demodectic rate of complete Demodex eradication in the treatment group $(11 / 27,40.74 \%)$ was slightly lower than that in the control group $(12 / 25,48 \%)$, but there was no significant difference between the two groups $\left(\chi^{2}=0.277, \mathrm{P}=0.598\right)$. Regarding the other ocular parameters, no significant difference was observed in the TBUT, meibum quality and MGE between the two groups $(\mathrm{P}<0.05)$. TTO group has a significantly improvement compared with Okra group in terms of SIT $(\mathrm{P}=0.035)$ and CFS $(\mathrm{P}=0.023)$. In conclusion, okra eyelid patch treatment is able to significantly eradicate ocular Demodex as well as markedly alleviate ocular symptoms. Due to causing less irritation than TTO, the okra eyelid patch may be more suitable for sensitive patients with Demodex blepharitis, such as the elderly and children. The study was registered as a clinical trial in the Chinese Clinical Trial Registry (ChiCTR) in November 2018 (registration no. ChiCTR-1,800,019,466).

\section{Introduction}

Demodex (class Arachnida, superorder Acariformes), a microscopic and elongated mite, is one of the most common ectoparasites in humans and may be present on the face and most other parts of the body $(1,2)$. The facial T-zone (forehead, cheeks and nose), meibomian glands (MGs) and follicles of eyelashes are the most common habitats of Demodex. Two parasitic species have been detected in humans, namely Demodex folliculorum and Demodex brevis. Eyelash follicles are the habitat of Demodex folliculorum, while Demodex brevis resides in the MGs and the sebaceous glands of the eyelids (3-5). Demodex infection gradually develops to Demodex blepharitis, which is characterized as chronic inflammation of the eyelid and MG and ultimately leads to MG dysfunction (MGD). MGD is the major cause of evaporated dry eye disease, characterized as tear film instability and chronic ocular surface inflammation $(6,7)$. Tea tree oil (TTO) may forcefully eradicate Demodex mites and alleviate ocular surface inflammation associated with Demodex blepharitis (8). Hence, TTO treatment is currently accepted as the most effective therapy for Demodex blepharitis. Although TTO has a certain therapeutic effect on Demodex blepharitis, the pungent odor of TTO may cause mild to moderate ocular irritation and discomfort at higher concentrations or with prolonged periods of exposure, especially to the elderly and children (6). 
Abelmoschus esculentus L., also known as okra, is a well-known tropical vegetable that is widely grown worldwide. In addition to serving as a food source, okra may serve as a traditional medicine to cure numerous diseases, such as dysentery and diarrhea (9). Due to abundant bioactive compounds, such as polysaccharides, flavonoids, polyphenols, caffeine and pectin in okra, the antibacterial, anti-inflammatory and immune regulatory effects of okra have been gradually proven (10-12). Okra has been applied widely in the cosmetics industry, where the safety and efficacy of okra have been gradually confirmed (13). Okra has been reported to exert anti-oxidative and anti-inflammatory effects by suppressing the Akt-mediated NF- $\mathrm{BB}$ pathway in a murine BV2 microglial cell line (14). Akt-mediated $N F-\kappa B$ pathway has been demonstrated to serve an important role in dry eye and blepharitis $(15,16)$. Due to the curative and medicinal effects of okra, it could be speculated that okra may confer therapeutic effects in patients with blepharitis and dry eye.

The present study was designed to determine the potential anti-demodectic and therapeutic effects of an okra eyelid patch on Demodex blepharitis in vivo and in vitro.

\section{Materials and methods}

Subjects. The present study was performed in accordance with the tenets of the Declaration of Helsinki and was approved by the Institutional Review Board of the Eye, Ear, Nose and Throat (EENT) Hospital of Fudan University. The study was registered as a clinical trial in the Chinese Clinical Trial Registry (ChiCTR) in November 2018 (registration no. ChiCTR-1,800,019,466). A total of 52 patients who experienced Demodex blepharitis were recruited from the EENT hospital between December 2018 and May 2019. Demodex blepharitis was confirmed by the total number of Demodex on epilated lashes under light microscopic examination. In brief, the procedure was as follows: A total of two lashes with cylindrical dandruff (CD) each from the upper and lower eyelids were epilated and mounted on glass slides; one drop of cedar oil was dripped on the bottom of each eyelash to dissolve the CD and to allow the embedded Demodex to migrate out, which occurs almost immediately; the total number of Demodex mites was counted by the same inspector (WL) under a light microscope (CX23; Olympus Corp.). If the number of Demodex mites was $\geq 3$, the patient was considered as Demodex-positive $(17,18)$. Furthermore, 'absolute Demodex eradication' was defined as complete Demodex eradication with the Demodex count reduced to zero after treatment $(19,20)$.

The inclusion criteria for the present study were Demodex blepharitis patients with Demodex-positive eyelashes aged 18-70 years who voluntarily participated in the experiment. As patients with certain ocular diseases (recurrent herpes simplex, ocular impairment associated with immune diseases) or receiving physiotherapy for blepharitis (intense pulsed light, baby shampoo, LipiFlow ${ }^{\circledR}$ and other Demodex treatments) in the last 6 months may have confounded the results, they were excluded from the study. Patients were also excluded if they had a related ocular surgery, including cataract surgery, trichiasis surgery or refractive surgery in the past 3 months. After the procedure and potential consequences of the treatments had been elaborately explained, informed consent was obtained from each participant prior to participation.
Treatments. A total of 52 participants were randomly divided into two groups using a random number table. The okra group $(n=27)$ received okra eyelid patch treatment $\left(\right.$ YourGa $^{\circledR}$; Shanghai YourGa Co., Ltd.; http://www.ganyanzheng.com/?_l=zh_CN). and the TTO group $(n=25)$ received TTO eye care patch treatment (YourGa; Shanghai YourGa Co., Ltd.; Fig. 1). The treatment was performed by participants at home every night before sleep for three months. The procedure was as follows: After the eyelid margin was cleaned by eye rinse (YourGa), the eyelid was heated to $42^{\circ} \mathrm{C}$ from the outside by a Moisture Chamber (YourGa) for $20 \mathrm{~min}$. Finally, eyelid patches (okra or TTO) were applied to both eyes for $15 \mathrm{~min}$ and then removed.

The order of examinations performed was as follows: Ocular surface disease index (OSDI), slit-lamp biomicroscopic examination, Schirmer I test (SIT), tear film break-up time (TBUT), MG assessment and corneal fluorescein staining (CFS). Each participant was subjected to the examinations three times, namely on the day prior to treatment and 1 and 3 months after treatment. All examinations were performed by a skilled researcher (WL) to reduce operational error. In addition, both eyes were examined and treated, but only the data of the right eye were analyzed.

OSDI. The OSDI questionnaire, a 12-item questionnaire with a scale of $0-100$, has been designed to rapidly evaluate ocular discomfort symptoms (e.g. soreness, light sensitiveness, blurred vision). The OSDI provides an assessment of vision-related dyspraxia (difficulty reading, driving, operating a computer and watching TV). There is a positive correlation between OSDI scores and the severity of ocular discomfort, with higher scores representing greater ocular discomfort (21). Prior to treatment, the OSDI questionnaire was completed by participants to set the baseline.

SIT. After a sterile dry strip (Jingming ${ }^{\circledR}$ ) was inserted into the lateral canthus of the lower eyelid away from the cornea for 5 min, the wetted length of the strip absorbed with tears was then measured to assess tear production. Potential SIT scores ranged from 0 to $30 \mathrm{~mm}$.

TBUT. A fluorescein strip (Jingming) moistened with preservative-free saline solution gently touched the central lower lid margin the patient was then requested to blink several times to ensure adequate coating of the complete corneal dye. After several natural blinks, the patient was required to rapidly open the eye and the time of eye opening was recorded as the starting point (time $=0 \mathrm{sec}$ ). TBUT was defined as the interval between the starting point and the first black spot appearing in the stained team film with a cobalt blue filter and slit lamp microscope. The test was repeated three times on each patient and the average TBUT was calculated (22).

$M G$ expressibility (MGE). A total of 5 consecutive MGE measurements of the central lower eyelid were assessed with an MG evaluator (MGE-1000; TearScience). The patient was instructed to look upwards and the lower eyelid gland orifices were gently cleaned with a cotton swab. The MG evaluator was held in position and pressure was maintained for 10-15 sec to evaluate whether each gland secreted or not. The MGE score was recorded according to the number of secretory glands: 0 , 


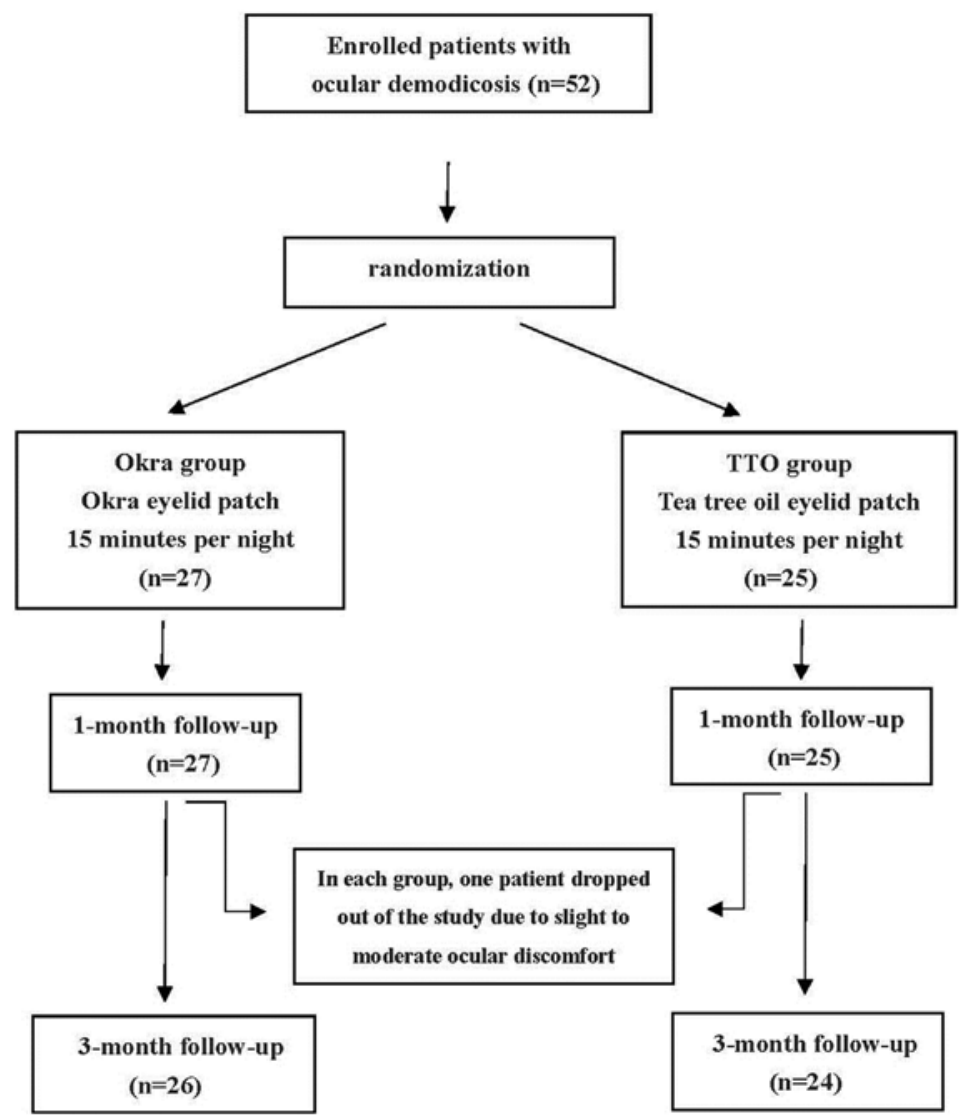

Figure 1. Flow chart indicating the total numbers of eyes enrolled, treated and observed during the follow-up period of the study. TTO, tea tree oil.

all five glands; 1 , three to four glands; 2 , one to two glands; and 3 , none of the glands (7).

Meibum quality. The meibum quality of eight central MGs of the upper eyelid was also assessed with the MG evaluator. The assessment procedure was the same as above. According to the secretion characteristics of each gland, the grade was ranked on a scale of 0 to 3 for each gland: 0 , clear liquid; 1 , colored/cloudy liquid; 2, cloudy with debris (granular); and 3, thick like toothpaste (total grade range, 0-24 scores) (7).

CFS. The inspection method of CFS was similar to that for the assessment of the TBUT. The cornea was divided into five zones (central, superior, temporal, nasal and inferior). Corneal epithelial injury was graded on a scale from 0 to 3 : 0 , no epithelial injury; $1,<30$ corneal punctate stains; $2,>30$ corneal punctate stains but not fusion; and 3 , fusion of corneal staining or ulcer. The total CFS score ranged from 0 to 15 (23).

Tolerance. During the treatment, any symptoms regardless of the cause were promptly recorded by the researcher (WL). Common symptoms may include eye redness, pain, allergy, irritation and foreign body sensation. Rare symptoms may involve blurred vision and acute ocular infection. If the participant requested withdrawal from the study due to discomfort, the study will be terminated immediately.

Survival time (ST) of Demodex. Written informed consents have been obtained from all patients before eyelashes have been extracted. After eyelashes have been extracted from the participants' eyelid in each group at room temperature, these eyelashes with demodex mites were instantly placed on a glass slide. A total of 60 mites were then divided randomly into three groups (20 mites in each group). Okra and TTO eyelid patch extractions were then dripped onto the glass with a micropipette respectively. Saline was used as the blank group. As Demodex is more vulnerable at an earlier stage of life, only adult Demodex with four pairs of well-developed legs and a stumpy body were tested (8). After the eyelash had been extracted from eyelid, the movements of the Demodex body and legs were observed immediately and continuously under a microscope at a magnification of $\mathrm{x} 40$. The $\mathrm{ST}$ was defined as the duration from the time-point of eyelid patch extraction dripped on the body to the cessation of movement (Fig. 2). The average ST of Demodex mites was compared among the control ( $\mathrm{n}=20$ mites), okra ( $\mathrm{n}=20$ mites) and TTO ( $\mathrm{n}=20$ mites) groups.

Statistical analysis. Randomization method was used to designate participants into Okra and TTO groups. Data were analyzed using SPSS v.17.0 software (SPSS Inc.). Categorical data (sex) between two groups were evaluated for statistical significance using the chi-square test. Continuous variables were presented as the mean \pm standard deviation. The normal distribution test (Kolmogorov-Smirnov) was performed to check whether the numerical variables were normally distributed. Data on age and ocular parameters between the two groups were evaluated for statistical significance using Student's t-test. The data 
A

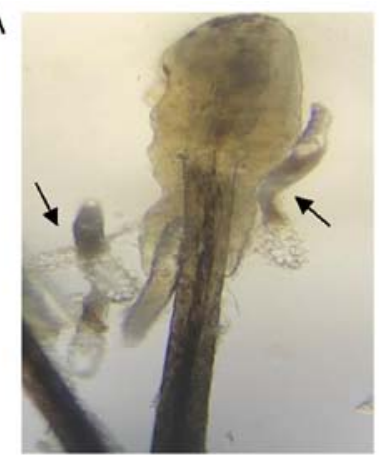

B

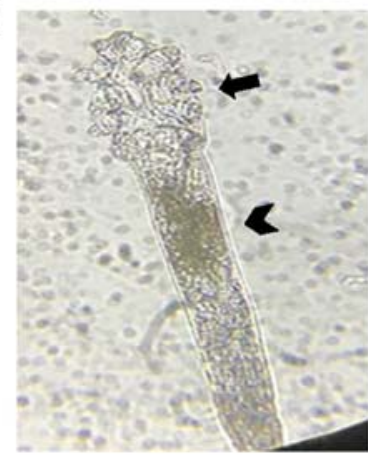

C

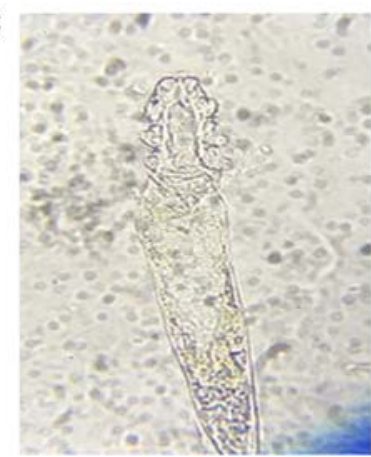

Figure 2. Microscopic examination of Demodex mites. (A) Several Demodex mites were detected under microscopic examination of the epilated lash (magnification, $\mathrm{x} 20$; thin arrows). (B and C) The one of the Demodex mite from (A) was photographed for live and dead status under a microscope (magnification, $\mathrm{x} 40$ ). (B) The Demodex mite was determined to be 'alive' if marked movements of the legs (thick arrow) and body (thick arrowhead) was present. (C) By contrast, the Demodex mite was determined to be 'dead' when movements of the leg ceased and the body became transparent.

at the baseline and at 1 and 3 months after treatment in the Okra group were homoscedastic and normally distributed and the differences between various time-points were analyzed by repeated-measures ANOVA followed by a least-significant differences test. The results are indicated as P-values, where $\mathrm{P}<0.05$ was considered to indicate a statistically significant difference. According to a previous study, the Demodex eradication rate of TTO therapy is $\sim 90 \%$ (20). In the present study, it was previously estimated that there would be a $25 \%$ relative difference between the Okra and the TTO groups (20), which meant that a sample size of 23 patients in each group was required to achieve a statistical power of $80 \%$ for a significance level of $0.25 \%$ with a two-tailed test.

\section{Results}

Patient baseline characteristics. A total of 27 participants underwent okra eyelid patch treatment (13 males and 14 females, aged $46.21 \pm 13.03$ years), while 25 participants received TTO eyelid patch treatment (13 males and 12 females, aged $40.54 \pm 10.39$ years). No significant differences in terms of sex $\left(c^{2}=0.103, P=0.749\right)$ and age $(\mathrm{P}=0.160)$ were determined between the two groups. There were no differences in the Demodex count, OSDI, meibum quality, MGE, SIT, TBUT and CFS between the okra and control groups prior to treatment $(\mathrm{P}>0.05$; Table I).

Demodex eradication and ocular parameters. The Demodex counts and ocular parameters at 1 and 3 months in the okra group were compared with those at baseline (Table II). Okra eyelid patch treatment can significantly eradicate Demodex mites from $10.15 \pm 4.53$ to $1.31 \pm 1.41$ on patients with ocular demodicosis after 3 months $(\mathrm{P}<0.01)$. The average OSDI score in the okra group decreased from baseline $(40.51 \pm 10.85)$ to the end of treatment (23.67 $\pm 10.71 ; \mathrm{P}<0.01)$. Other ocular parameters, including SIT, TBUT, CFS, meibum quality and MGE, were also compared before and after 1 and 3 months of treatment, respectively. Statistically significant improvements in TBUT ( $\mathrm{P}=0.007)$, CFS $(\mathrm{P}<0.01)$ and meibum quality $(\mathrm{P}<0.01)$ have been observed in Okra group by 3 months. Blepharitis of 13 patients was observed to be improved from slight to obvious degrees after Okra eyelid treatment. Among these patients, the sign of eyelid margin in one patient who was improved markedly (Fig. 3).
The Demodex counts and ocular parameters at 1 and 3 months of treatment were also compared between the okra and TTO groups (Table III). The average Demodex count in the okra group had decreased by $-8.85 \pm 3.84$ after 3 months of treatment, whilst the Demodex count in the TTO group decreased $-9.36 \pm 6.03$ after 3 months of treatment $(\mathrm{P}=0.716)$. There was no significant difference in the anti-demodectic effects between the two groups at 3 months $(\mathrm{P}=0.716)$. Compared with that in the TTO group, the anti-demodectic effect of okra was slightly milder at 1 month, but no significant difference was observed between the two treatments at 1 and 3 months. The absolute Demodex eradication rate in the okra group $(11 / 27,40.74 \%)$ was slightly lower than that in the TTO group $(12 / 25,48 \%)$. The improvements in each of ocular parameters between okra and TTO groups did not exhibit significant difference, except for SIT $(\mathrm{P}=0.035)$ and CFS $(\mathrm{P}=0.023)$ at 3 months.

Tolerance. Any ocular discomfort, including allergic reaction, irritation and pruritus, acute ocular infection and visual acuity loss, was recorded during treatment. No allergic reactions or acute ocular infections were reported during treatment in either group. Of the 27 participants in the okra group, $26(96.3 \%)$ did not report any adverse events or tolerability issues during the treatment period. However, one participant (3.7\%) reported transient ocular pruritus and discomfort following application of the okra eyelid patch close to the eyelash margin and surrounding skin. This participant was then dropped out of the study and lost to follow-up. Of the 25 patients in the TTO group, four $(16 \%)$ reported slight to moderate irritation with conjunctival congestion. Among these four participants, one was subsequently lost to follow-up due to dropping out.

ST of Demodex. The average ST of Demodex in the different groups is provided in Fig. 4. The average ST in the okra group was $115.25 \mathrm{~min}$, which was significantly lower compared with the average ST of $378.75 \mathrm{~min}$ in the blank group $(\mathrm{P}<0.01)$. Compared with that in the TTO group (106.7 min), the ST in the okra group was slightly but significantly longer $(\mathrm{P}=0.042)$.

\section{Discussion}

Increased attention has been paid to Demodex blepharitis by ophthalmologists in the past two decades. Several pathogenic 
Table I. Comparison of demographic data, baseline data between the okra and TTO treatment groups.

\begin{tabular}{lccc}
\hline Item & Okra $(\mathrm{n}=27)$ & TTO (n=25) & P-value \\
\hline Age (years) & $46.21 \pm 13.03$ & $40.54 \pm 10.39$ & 0.160 \\
Females/males & $14 / 13$ & $12 / 13$ & 0.749 \\
Demodex mites & $10.15 \pm 4.53$ & $11.24 \pm 5.88$ & 0.455 \\
OSDI score & $40.51 \pm 10.85$ & $35.86 \pm 12.77$ & 0.162 \\
Meibum quality score & $8.56 \pm 4.26$ & $6.68 \pm 4.72$ & 0.138 \\
MGE & $1.15 \pm 0.66$ & $1.44 \pm 0.51$ & 0.082 \\
SIT (mm/5 min) & $9.63 \pm 5.13$ & $7.20 \pm 6.70$ & 0.147 \\
TBUT (sec) & $4.59 \pm 1.74$ & $5.12 \pm 1.88$ & 0.298 \\
CFS & $2.22 \pm 1.60$ & $1.40 \pm 1.68$ & 0.077
\end{tabular}

Values are expressed as the mean \pm standard deviation or $\mathrm{n}$. CFS, corneal fluorescein staining; MGE, Meibomian gland expressibility; OSDI, ocular surface disease index; SIT, Schirmer I test; TTO, tea tree oil; TBUT, tear break-up time.

Table II. Variation in the parameters from baseline to 1 and 3 months after treatment in the Okra group.

\begin{tabular}{lccrrrr}
\hline Item & Prior to treatment & 1 month & 3 months & F & P1 \\
\hline Demodex mites & $10.15 \pm 4.53$ & $3.26 \pm 2.03$ & $1.30 \pm 1.41$ & 125.55 & $<0.01$ & $<0.01$ \\
OSDI score & $40.51 \pm 10.85$ & $29.55 \pm 12.15$ & $23.67 \pm 10.71$ & 29.74 & $<0.01$ & $<0.01$ \\
Meibum quality score & $8.56 \pm 4.26$ & $5.85 \pm 4.29$ & $5.48 \pm 4.08$ & 16.39 & $<0.01$ & $<0.01$ \\
MGE & $1.15 \pm 0.66$ & $0.93 \pm 0.62$ & $1.04 \pm 0.71$ & 1.44 & 0.056 & 0.449 \\
SIT & $9.63 \pm 5.13$ & $10.74 \pm 4.71$ & $11.22 \pm 4.53$ & 3.03 & 0.119 & 0.014 \\
TBUT (sec) & $4.59 \pm 1.74$ & $5.56 \pm 1.50$ & $5.96 \pm 1.58$ & 12.74 & 0.006 & $<0.01$ \\
CFS & $2.22 \pm 1.60$ & $1.11 \pm 1.12$ & $0.78 \pm 0.93$ & 43.54 & $<0.01$ & $<0.01$ \\
\hline
\end{tabular}

Values are expressed as the mean \pm standard deviation. P-values: P1, pretreatment vs. 1 month; P2, pretreatment vs. 3 months. F, variance values among three groups; CFS, corneal fluorescein staining; MGE, Meibomian gland expressibility; OSDI, ocular surface disease index; SIT, Schirmer I test; TBUT, tear break-up time.
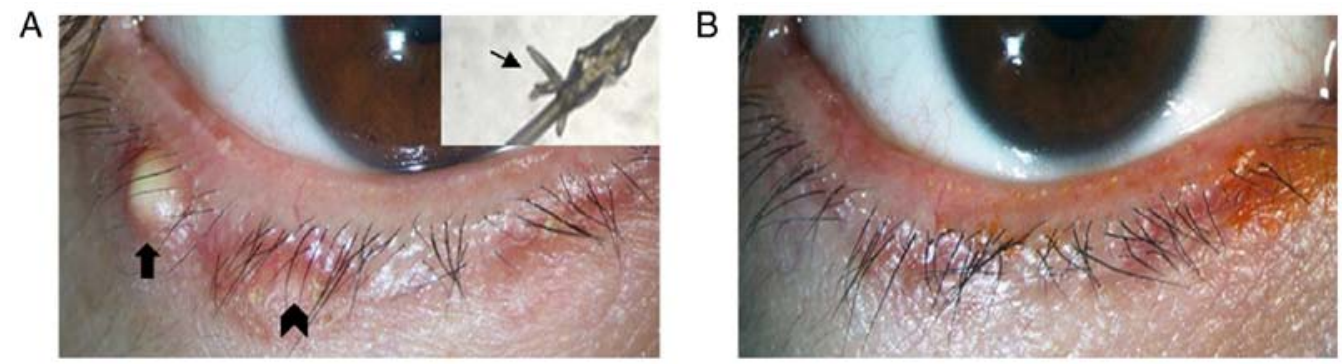

Figure 3. Photographs of the palpebral margin of a representative case (male, 40 years old) in the okra group prior to and after treatment. (A) Prior to okra eyelid treatment, Demodex was strongly positive (thin arrowhead), with blepharitis (thick arrowhead) and local hordeolum (thick arrow; magnification, x16). (B) After 3 months of okra treatment, Demodex had been eradicated and symptoms had significantly improved.

mechanisms of Demodex blepharitis have been postulated in previous studies. First, Demodex mites may significantly damage the habitat where they live by continuous movement and invasion. Furthermore, Demodex mites may block the hair follicles and sebaceous ducts mechanically to induce epithelial hyperplasia and hyperkeratinization, while debris or waste from Demodex mites may elicit inflammatory responses or an innate immune response (24). In addition to the mechanisms described above, the pathogenic role of other microbial infections, including Streptococci, Staphylococci, Propionibacterium acnes associated with Demodex infestation that leads to MGD and dry eye has also gained increasing attention.

Different TTO products are now widely applied in Demodex blepharitis treatments. A weekly eyelid scrub with $50 \%$ TTO proved successful in eradicating ocular Demodex infestation and Demodex counts as low as zero have been confirmed in the majority of patients after 4 weeks of 
Table III. Variation in the parameters from baseline to 1 and 3 months after treatment compared between the two groups.

\begin{tabular}{|c|c|c|c|c|c|c|}
\hline \multirow[b]{2}{*}{ Item } & \multicolumn{3}{|c|}{1 month } & \multicolumn{3}{|c|}{3 months } \\
\hline & Okra & TTO & $\mathrm{P} 1$ & Okra & TTO & $\mathrm{P} 2$ \\
\hline Demodex mite eradication & $-6.89 \pm 3.24$ & $-7.84 \pm 6.24$ & 0.489 & $-8.85 \pm 3.84$ & $-9.36 \pm 6.03$ & 0.716 \\
\hline OSDI score & $-8.65 \pm 11.49$ & $-12.40 \pm 9.53$ & 0.627 & $-16.84 \pm 10.17$ & $-17.44 \pm 16.05$ & 0.873 \\
\hline Meibum quality score & $-2.70 \pm 2.30$ & $-1.56 \pm 2.45$ & 0.089 & $-3.07 \pm 3.15$ & $-2.48 \pm 3.42$ & 0.517 \\
\hline MGE & $-0.22 \pm 0.58$ & $-0.04 \pm 0.20$ & 0.141 & $-0.11 \pm 0.75$ & $-0.32 \pm 0.63$ & 0.284 \\
\hline $\mathrm{SIT}(\mathrm{mm} / 5 \mathrm{~min})$ & $1.11 \pm 3.58$ & $-0.36 \pm 1.68$ & 0.067 & $1.59 \pm 3.13$ & $0.04 \pm 1.81$ & 0.035 \\
\hline TBUT (sec) & $0.96 \pm 1.68$ & $0.16 \pm 1.72$ & 0.095 & $1.37 \pm 1.47$ & $0.76 \pm 1.83$ & 0.190 \\
\hline CFS & $-1.11 \pm 0.89$ & $-0.44 \pm 0.87$ & 0.008 & $-1.44 \pm 0.93$ & $-0.72 \pm 1.28$ & 0.023 \\
\hline
\end{tabular}

Values are expressed as the mean \pm standard deviation. P-values: P1, pretreatment vs. 1 month; P2, pretreatment vs. 3 months. F, variance values among three groups; TTO, tea tree oil; CFS, corneal fluorescein staining; MGE, Meibomian gland expressibility; OSDI, ocular surface disease index; SIT, Schirmer I test; TBUT, tear break-up time.

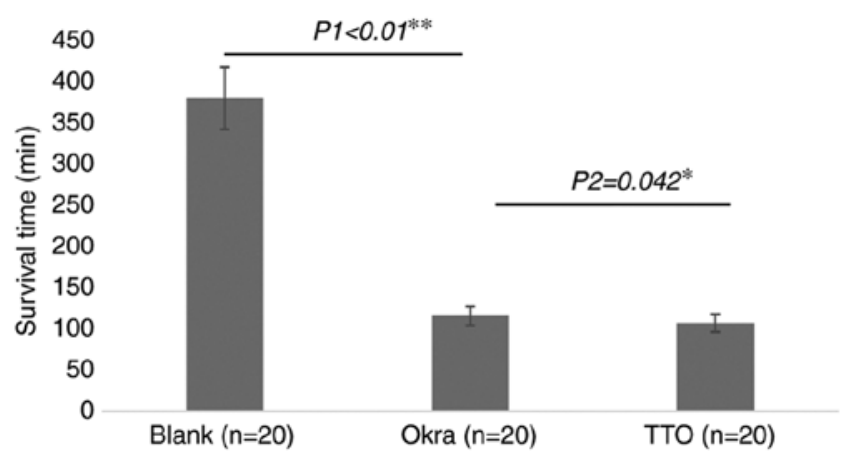

Figure 4. Survival time of Demodex mites under different treatments. Each bar represents the mean survival time and error bars represent the standard deviation. ${ }^{*} \mathrm{P}<0.05$, Okra vs. TTO $;{ }^{* *} \mathrm{P}<0.01$, Blank vs. Okra. TTO, tea tree oil.

treatment (25-27). The present study indicated that okra had a similar effect to that of TTO in terms of Demodex eradication on the eyelids of patients, and furthermore, it significantly shortened the survival time of Demodex in vitro. Therefore, okra is expected to be developed as a potential treatment for Demodex blepharitis.

As TTO may exert antibacterial, antifungal and anti-inflammatory actions, its therapeutic effects may not be attributed to killing Demodex mites only; TTO also possesses anti-inflammatory and bacterial colonization reduction properties. In previous studies, TTO demonstrated a strong effect on Demodex eradication and among a total of 15 major active components in TTO, terpinen-4-ol has been identified as the most potent ingredient $(8,26)$.

Regarding bacterial infection, okra fruit has a high tannin content that may abolish several common types of bacteria, including several Gram-positive and Gram-negative bacteria (28). Furthermore, Lengsfeld et al (29) reported that $H$. pylori adhesion on human stomach sections was almost completely inhibited by fresh okra fruit juice. In addition to its antibacterial effects, a number of studies suggested that crude okra acts as an immunomodulator with both immune-stimulatory and immunosuppressive activities (30). Okra is also able to act as an immune modulator by activating phagocytes to produce more proinflammatory cytokines (e.g. TNF- $\alpha$ and IL-17) to improve the host defense against various bacteria. Among the medicinal herbs, okra is an important plant that is widely distributed worldwide; furthermore, okra is a Chinese medicine and is less pungent than TTO, resulting in minor toxicity and few side effects (31).

Among antiparasitic remedies, polysaccharides from different sources have become a prime research topic. Volatile oils from certain Chinese crude medicines with abundant polysaccharides exerted the most potent effect among various Demodex blepharitis treatments (32). Apart from this, sulfated polysaccharides from algae were reported to serve as an alternative to heparin in the treatment of leishmaniasis to significantly reduce the count of the promastigotes of L. amazonensis in a dose-dependent manner (33). The possible anti-demodectic mechanism of polysaccharides is to regulate the host's immune system by activating immune cells such as lymphocytes, macrophages and natural killer cells. Furthermore, the attachment between host and parasite may be attenuated (34). Okra contains an abundance of polysaccharides, which are considered to be responsible for effective Demodex eradication.

In addition, okra, which contains an abundance of flavonoids, polyphenol and vitamin $\mathrm{C}$, may act as an antioxidant and anti-inflammatory agent with low toxicity and few side effects $(35,36)$. The antioxidant activity of methanolic seed extracts of okra has been reported in several studies, revealing antioxidant activity of okra seed extracts under different conditions $(37,38)$. Furthermore, the reactive oxygen species (ROS) oxidation pathway has been confirmed to involve in the pathogenesis of dry eye disease, whereas okra may interfere with dry eye by inhibiting the ROS oxidation pathway to alleviate ocular discomfort $(39,40)$.

Similar to Demodex blepharitis, rosacea is a characteristic cutaneous dermatosis due to the presence of multiple small, dome-shaped erythematous papules and papulopustules and Demodex mites may also have a vital role in its pathogenesis $(41,42)$. Certain studies have suggested that rosacea remedies, including antibacterial, anti-inflammatory and immune regulation treatments, have proven curative 
effects (43). A number of previous studies suggested that antibiotic treatments, such as oral antibiotics, including tetracycline or topical metronidazole, may obviously alleviate skin inflammation and symptoms of rosacea. Superantigens produced by Streptococci and Staphylococci may have a role in the induction of rosacea (43). Hence, researchers should also pay more attention to the role of microorganisms in the treatment of Demodex infestation $(44,45)$. Due to the reported anti-inflammatory and antioxidant effects of okra $(28,37)$, it could be speculated that the symptoms of Demodex blepharitis may be improved by these compounds. In addition, it has been proposed that Demodex may be eradicated by changing the living environment of Demodex and reducing the colonization of related bacteria in MG in view of the antibacterial, anti-inflammatory and immunoregulatory effects of okrae 30,36). In the present study, okra caused less irritation than TTO, was more comfortable and resulted in good compliance. In conclusion, okra may be a potential remedy for the treatment of Demodex blepharitis and MGD.

Due to the limitation of the small number of participants in the present study, the efficacy of okra eyelid patches for Demodex blepharitis requires further verification in a larger cohort. Although the anti-demodectic effects of the okra eyelid patch in Demodex blepharitis are explicit, the mechanisms of the anti-demodectic effects of okra's remain to be fully elucidated. Future studies are required to clarify the anti-demodectic mechanism of okra.

In conclusion, the okra eyelid patch effectively eradicated Demodex mites both in patients and in vitro and its application was associated with reduced ocular discomfort. The okra eyelid patch presented superior ocular tolerance and may be more comfortable to use than the TTO eyelid patch.

\section{Acknowledgements}

Not applicable.

\section{Funding}

The present study was sponsored by Shanghai Sailing Program (grant. no. 19YF1405800).

\section{Availability of data and materials}

The datasets used and/or analyzed during the present study are available from the corresponding author on reasonable request.

\section{Authors' contributions}

LG participated in the project design, sample size calculation and revision of the manuscript. WL was responsible for the enrolment and follow-up of patients and participated in performing the statistical analysis. WL also drafted the manuscript. Both authors confirm the authenticity of the raw data and read and approved the final manuscript.

\section{Ethics approval and consent to participate}

The present study was strictly performed on the basis of the Declaration of Helsinki for research involving human participants and was approved by the Ethics Committee of the EENT Hospital of Fudan University (Shanghai, China). After the experimental details and potential benefits and risks were explained, written informed consent was obtained from all participants prior to the examination and treatment.

\section{Patient consent for publication}

Oral consent from the patient whose lid margin and Demodex photographs are displayed in Fig. 3 was obtained prior to publication.

\section{Competing interests}

The authors declare that they have no competing interests.

\section{References}

1. Basta-Juzbasić A, Subić JS and Ljubojević S: Demodex folliculorum in development of dermatitis rosaceiformis steroidica and rosacea-related diseases. Clin Dermatol 20: 135-140, 2002.

2. Wesolowska M, Knysz B, Reich A, Blazejewska D, Czarnecki M, Gladysz A, Pozowski A and Misiuk-Hojlo M: Prevalence of Demodex spp. in eyelash follicles in different populations. Arch Med Sci 10: 319-324, 2014.

3. Zhang XB, Ding $\mathrm{YH}$ and $\mathrm{He} \mathrm{W}$ : The association between Demodex infestation and ocular surface manifestations in meibomian gland dysfunction. Int J Ophthalmol 11: 589-592, 2018.

4. Fromstein SR, Harthan JS, Patel J and Opitz DL: Demodex blepharitis: Clinical perspectives. Clin Optom (Auckl) 10: 57-63, 2018.

5. Luo X, Li J, Chen C, Tseng S and Liang L: Ocular demodicosis as a potential cause of ocular surface inflammation. Cornea 36 (Suppl 1): S9-S14, 2017.

6. Bron AJ, de Paiva CS, Chauhan SK, Bonini S, Gabison EE, Jain S, Knop E, Markoulli M, Ogawa Y, Perez V, et al: TFOS DEWS II pathophysiology report. Ocul Surf 15: 438-510, 2017.

7. Tomlinson A, Bron AJ, Korb DR, Amano S, Paugh JR, Pearce EI, Yee R, Yokoi N, Arita R and Dogru M: The international workshop on meibomian gland dysfunction: Report of the diagnosis subcommittee. Invest Ophthalmol Vis Sci 52: 2006-2049, 2011.

8. Gao YY, Di Pascuale MA, Li W, Baradaran-Rafii A, Elizondo A, Kuo CL, Raju VK and Tseng SC: In vitro and in vivo killing of ocular Demodex by tea tree oil. Br J Ophthalmol 89: 1468-1473, 2005.

9. Doreddula SK, Bonam SR, Gaddam DP, Desu BS, Ramarao N and Pandy V: Phytochemical analysis, antioxidant, antistress, and nootropic activities of aqueous and methanolic seed extracts of ladies finger (Abelmoschus esculentus L.) in mice. ScientificWorldJournal 2014: 519848, 2014.

10. Adelakun OE, Oyelade OJ, Ade-Omowaye BI, Adeyemi IA and Van de Venter M: Chemical composition and the antioxidative properties of nigerian okra seed (Abelmoschus esculentus Moench) flour. Food Chem Toxicol 47: 1123-1126, 2009.

11. Liao H, Dong W, Shi X, Liu H and Yuan K: Analysis and comparison of the active components and antioxidant activities of extracts from Abelmoschus esculentus L. Pharmacogn Mag 8: 156-161, 2012.

12. Cho CW, Han CJ, Rhee YK, Lee YC, Shin KS, Shin JS, Lee KT and Hong HD: Cheonggukjang polysaccharides enhance immune activities and prevent cyclophosphamide-induced immunosuppression. Int J Biol Macromol 72: 519-525, 2015.

13. Durazzo A, Lucarini M, Novellino E, Souto EB, Daliu P and Santini A: Abelmoschus esculentus (L.): Bioactive components beneficial properties-focused on antidiabetic role-for sustainable health applications. Molecules 24: 38, 2018.

14. Mairuae N, Cheepsunthorn P, Cheepsunthorn CL and Tongjaroenbuangam W: Okra (Abelmoschus esculentus Linn) inhibits lipopolysaccharide-induced inflammatory mediators in BV2 microglial cells. Trop J Pharm Res 16: 1285, 2017.

15. Zhang X, Yin Y, Yue L and Gong L: Selective serotonin reuptake inhibitors aggravate depression-associated dry eye via activating the NF- $\kappa$ B pathway. Invest Ophthalmol Vis Sci 60: 407-419, 2019. 
16. Yang FM, Fan D, Yang XQ, Zhu FH, Shao MJ, Li Q, Liu YT, Lin ZM, Cao SQ, Tang W, et al: The artemisinin analog SM934 alleviates dry eye disease in rodent models by regulating TLR4/NF- $\kappa$ B/NLRP3 signaling. Acta Pharmacol Sin: Aug 3, 2020 (Epub ahead of print).

17. Gao YY, Di Pascuale MA, Li W, Liu DT, Baradaran-Rafii A, Elizondo A, Kawakita T, Raju VK and Tseng SC: High prevalence of Demodex in eyelashes with cylindrical dandruff. Invest Ophthalmol Vis Sci 46: 3089-3094, 2005.

18. Kheirkhah A, Blanco G, Casas V and Tseng SC: Fluorescein dye improves microscopic evaluation and counting of demodex in blepharitis with cylindrical dandruff. Cornea 26: 697-700, 2007.

19. Salem DA, El-Shazly A, Nabih N, El-Bayoumy Y and Saleh S: Evaluation of the efficacy of oral ivermectin in comparison with ivermectin-metronidazole combined therapy in the treatment of ocular and skin lesions of Demodex folliculorum. Int J Infect Dis 17: e343-e347, 2013.

20. Zhang X, Song $N$ and Gong L: Therapeutic effect of intense pulsed light on ocular demodicosis. Curr Eye Res 44: 250-256, 2019.

21. Schiffman RM, Christianson MD, Jacobsen G, Hirsch JD and Reis BL: Reliability and validity of the ocular surface disease index. Arch Ophthalmol 118: 615-621, 2000.

22. Yokoi N, Georgiev GA, Kato H, Komuro A, Sonomura Y, Sotozono C, Tsubota K and Kinoshita S: Classification of fluorescein breakup patterns: A novel method of differential diagnosis for dry eye. Am J Ophthalmol 180: 72-85, 2017.

23. Lemp MA: Report of the national eye institute/industry workshop on clinical trials in dry eyes. CLAO J 21: 221-232, 1995

24. Bevins CL and Liu FT: Rosacea: Skin innate immunity gone awry? Nat Med 13: 904-906, 2007.

25. Gao YY, Di Pascuale MA, Elizondo A and Tseng SC: Clinical treatment of ocular demodecosis by lid scrub with tea tree oil. Cornea 26: 136-143, 2007.

26. Tighe S, Gao YY and Tseng SC: Terpinen-4-ol is the most active ingredient of tea tree oil to kill Demodex mites. Transl Vis Sci Technol 2: 2, 2013

27. Koo H, Kim TH, Kim KW, Wee SW, Chun YS and Kim JC: Ocular surface discomfort and Demodex: Effect of tea tree oil eyelid scrub in Demodex blepharitis. J Korean Med Sci 27: 1574-1579, 2012

28. Messing J, Thöle C, Niehues M, Shevtsova A, Glocker E, Borén T and Hensel A: Antiadhesive properties of Abelmoschus esculentus (Okra) immature fruit extract against Helicobacter pylori adhesion. PLoS One 9: e84836, 2014.

29. Lengsfeld C, Titgemeyer F, Faller G and Hensel A: Glycosylated compounds from okra inhibit adhesion of Helicobacter pylori to human gastric mucosa. J Agric Food Chem 52: 1495-1503, 2004.

30. Wahyuningsih SPA, Pramudya M, Putri IP, Winarni D, Savira NII and Darmanto W: Crude polysaccharides from okra pods (Abelmoschus esculentus) grown in indonesia enhance the immune response due to bacterial infection. Adv Pharmacol Sci 2018: 8505383, 2018.

31. Polito L, Bortolotti M, Maiello S, Battelli MG and Bolognesi A: Plants producing ribosome-inactivating proteins in traditional medicine. Molecules 21: 1560, 2016.
32. Liu JX, Sun YH and Li CP: Volatile oils of Chinese crude medicines exhibit antiparasitic activity against human Demodex with no adverse effects in vivo. Exp Ther Med 9: 1304-1308, 2015.

33. Lehnhardt Pires C, Rodrigues SD, Bristot D, Gaeta HH, de Oliveira Toyama D, Lobo Farias WR and Toyama MH: Evaluation of macroalgae sulfated polysaccharides on the leishmania (L.) amazonensis promastigote. Mar Drugs 11: 934-943, 2013.

34. Jiang MH, Zhu L and Jiang JG: Immunoregulatory actions of polysaccharides from Chinese herbal medicine. Expert Opin Ther Targets 14: 1367-1402, 2010.

35. Shui G and Peng LL: An improved method for the analysis of major antioxidants of hibiscus esculentus Linn. J Chromatogr A 1048: 17-24, 2004

36. Chen H, Jiao H, Cheng Y, Xu K, Jia X, Shi Q, Guo S, Wang M, Du L and Wang F: In vitro and in vivo immunomodulatory activity of okra (Abelmoschus esculentus L.) polysaccharides. J Med Food 19: 253-265, 2016.

37. Hu L, Yu W, Li Y, Prasad N and Tang Z: Antioxidant activity of extract and its major constituents from okra seed on rat hepatocytes injured by carbon tetrachloride. Biomed Res Int 2014: 341291,2014

38. Khomsug P, Thongjaroe W, Pakdeenaro N, Suttajit M and Chantirati P: Antioxidative activities and phenolic content of extracts from okra (Abelmoschus esculentus L.). Res J Biol Sci 5: 310-313, 2010.

39. Chi W, Hua X, Chen X, Bian F, Yuan X, Zhang L, Wang X, Chen D, Deng R, Li Z, et al: Mitochondrial DNA oxidation induces imbalanced activity of NLRP3/NLRP6 inflammasomes by activation of caspase- 8 and BRCC 36 in dry eye. J Autoimmun 80: 65-76, 2017.

40. Saravanan S, Pandikumar P, Pazhanivel N, Paulraj MG and Ignacimuthu S: Hepatoprotective role of Abelmoschus esculentus (Linn.) moench., on carbon tetrachloride-induced liver injury. Toxicol Mech Methods 23: 528-536, 2013.

41. Powell FC: Clinical practice. Rosacea. N Engl J Med 352: 793-803, 2005

42. Li J, O'Reilly N, Sheha H, Katz R, Raju VK, Kavanagh K and Tseng SC: Correlation between ocular Demodex infestation and serum immunoreactivity to bacillus proteins in patients with facial rosacea. Ophthalmology 117: 870-877.e1, 2010.

43. Baldwin HE: Diagnosis and treatment of rosacea: State of the art. J Drugs Dermatol 11: 725-730, 2012.

44. Huang CN, Wang CJ, Lee YJ and Peng $\mathrm{CH}$ : Active subfractions of Abelmoschus esculentus substantially prevent free fatty acid-induced $\beta$ cell apoptosis via inhibiting dipeptidyl peptidase-4. PLoS One 12: e0180285, 2017.

45. Mollick MMR, Bhowmick B, Mondal D, Maity D, Rana D, Dash SK, Chattopadhyay S, Roy S, Sarkar J, Acharya K, et al: Anticancer (in vitro) and antimicrobial effect of gold nanoparticles synthesized using Abelmoschus esculentus (L.) pulp extract via a green route. RSC Adv 4: 37838-37848, 2014.

This work is licensed under a Creative Commons Attribution-NonCommercial-NoDerivatives 4.0 International (CC BY-NC-ND 4.0) License. 